\title{
Oral Activated Charcoal Adsorbent (AST-120) Ameliorates Chronic Kidney Disease-Induced Intestinal Epithelial Barrier Disruption
}

\author{
Nosratola D. Vaziria Jun Yuan ${ }^{a}$ Mahyar Khazaelia Yuichi Masuda ${ }^{b}$ \\ Hirohito Ichii ${ }^{b}$ Shuman Liu ${ }^{a}$ \\ ${ }^{a}$ Division of Nephrology and Hypertension, and ${ }^{b}$ Department of Surgery, University of California, Irvine, Calif., USA
}

\author{
Key Words \\ Epithelial tight junction - Endotoxin · Activated charcoal . \\ End-stage renal disease - Uremia
}

\begin{abstract}
Background: Chronic kidney disease (CKD) impairs intestinal barrier function which by allowing influx of noxious products causes systemic inflammation. We have recently shown that intestinal barrier dysfunction in CKD is due to degradation of epithelial tight junction (TJ) which is, in part, mediated by influx of urea and its conversion to ammonia by microbial urease. We hypothesized that by adsorbing urea and urea-derived ammonia, oral activated charcoal (AST-120) may ameliorate CKD-induced intestinal epithelial barrier disruption and systemic inflammation. Methods: Rats were randomized to the CKD or control groups. The CKD group was fed a chow containing $0.7 \%$ adenine for 2 weeks. They were then randomized to receive a chow with or without AST-120 (4 g/kg/day) for 2 weeks. Rats consuming regular diet served as controls. Animals were then euthanized, colons were removed and processed for Western blot and immunohistology, and plasma was used to measure endotoxin and oxidative and inflammatory markers. Results: Compared with the controls, the untreated CKD rats showed elevated plasma endotoxin, IL-6, TNF-a, MCP-1, CINC-3, L-selectin, ICAM-1, and malondialdehyde, and depletions of
\end{abstract}

colonic epithelial TJ proteins, claudin-1, occludin, and ZO1. Administration of AST-120 resulted in partial restoration of the epithelial TJ proteins and reduction in plasma endotoxin and markers of oxidative stress and inflammation. Conclusions: CKD animals exhibited depletion of the key protein constituents of the colonic epithelial TJ which was associated with systemic inflammation, oxidative stress and endotoxemia. Administration of AST-120 attenuated uremia-induced disruption of colonic epithelial TJ and the associated endotoxemia, oxidative stress and inflammation.

Copyright $\odot 2013$ S. Karger AG, Basel

\section{Introduction}

In addition to serving as the vehicle for absorption of nutrients and excretion of waste, the gastrointestinal tract serves as a barrier against entry of the microorganism, microbial toxins, digestive enzymes, degraded food products, antigens, and other harmful luminal contents into the body's internal environment. Impairment of the intestinal epithelial barrier structure and function leads to local and systemic inflammation by allowing the influx of these products in the internal milieu. The intestinal barrier encompasses the epithelial cells and the apical junctional complex which seals the gap between the adjacent cells [1]. The apical junctional complex consists of the

\section{KARGER}

E-Mail karger@karger.com

www.karger.com/ajn
(C) 2013 S. Karger AG, Basel

0250-8095/13/0376-0518\$38.00/0
N.D. Vaziri, MD, MACP

Division of Nephrology and Hypertension, University of California Irvine Medical Center, Suite 400, City Tower, 101 The City Drive Orange, CA 92868 (USA)

E-Mail ndvaziri@uci.edu 
tight junction (TJ) which is the most luminal component of the complex and the subjacent adherens junction. The TJ consists of the transmembrane, cytosolic, and perijunctional proteins. The transmembrane TJ proteins include the occludin and claudin protein families which consist of adhesive proteins that connect the plasma membranes of the adjacent cells forming the barrier to diffusion of fluids and solutes. The cytosolic proteins include the zonula occludens ( $\mathrm{ZO}$ ) protein family which simultaneously bind the intracellular domains of occludin and claudin and the perijunctional actin-myosine ring. The perijunctional proteins consist of the actin and myosin complex which regulate paracellular permeability by modulating the structure and function of the TJ [2].

Chronic kidney disease (CKD) is associated with oxidative stress and systemic inflammation which play a central role in the pathogenesis of cardiovascular disease and numerous other morbidities in this population [3-5]. There is increasing evidence that point to the intestinal barrier dysfunction and its contribution to the systemic inflammation in patients and animals with advanced renal disease [6]. In this context, studies conducted in the uremic humans and animals have revealed increased intestinal permeability to large molecular weight polyethylene glycols $[7,8]$ and studies in uremic rats have demonstrated penetration of bacteria across the intestinal wall and their detection in the mesenteric lymph nodes [9]. In addition, histological examination of the gastrointestinal tissues have revealed presence of chronic inflammation throughout the gastrointestinal tract in hemodialysis population [10], and several studies have revealed presence of endotoxemia in the absence of infection in uremic patients [11-13]. Taken together, these observations provided strong evidence for increased intestinal permeability and barrier dysfunction in patients and animals with advanced CKD. However, until recently the nature and the mechanisms by which uremia increased intestinal epithelial permeability were not known. In a recent study, we found heavy losses of the key protein constituents of colonic epithelial TJ in rats with CKD [14]. The depletion of intestinal epithelial $\mathrm{TJ}$ which is the principal barrier against influx of endotoxin and other noxious luminal contents into the systemic circulation, shown in that study unraveled the source of endotoxemia which is commonly present and is a major cause of inflammation in CKD [11]. In a subsequent study [15], we asked whether $\mathrm{CKD}$-induced disruption of the intestinal epithelial barrier is caused by retained uremic toxins or metabolites and if so whether they can be removed by dialysis. To address this question, we compared the effects of pre- and post-hemodialysis plasma samples from ESRD patients and plasma from healthy controls on the epithelial barrier function and structure in cultured human colonic epithelial cells seeded on Transwell plates to form a polarized, impermeable monolayer that simulates characteristics of intestinal epithelium in vivo. The study showed that compared with the control plasma, incubation in media containing pre-dialysis plasma from ESRD patients caused a marked drop in transepithelial electrical resistance denoting increased epithelial permeability. This was accompanied by significant loss of the TJ proteins. The severity of the epithelial barrier damage and dysfunction was significantly less in cells exposed to the post-dialysis than pre-dialysis plasma pointing to the role of some dialyzable uremic retained product(s) [15]. In a subsequent study, we tested the hypothesis that the breakdown of the intestinal barrier in CKD is at least in part mediated by the heavy influx of urea into the gastrointestinal tract, its conversion by microbial urease to ammonia $\left[\mathrm{CO}\left(\mathrm{NH}_{2}\right) 2+\mathrm{H}_{2} \mathrm{O} \rightarrow \mathrm{CO}_{2}+2 \mathrm{NH}_{3}\right]$ and formation of ammonium hydroxide $\left[\mathrm{NH}_{3}+\mathrm{H}_{2} \mathrm{O} \rightarrow \mathrm{NH}_{4} \mathrm{OH}\right]$, a caustic compound which can dissolve the TJ proteins. To this end, we incubated the fully polarized human enterocytes in the culture media containing clinically relevant concentrations of urea. To simulate the presence of microbial flora, we repeated the experiments by adding urea plus urease to the culture media. The study revealed that at clinically relevant concentrations, urea caused a concentration-dependent fall in transepithelial electrical resistance and a significant reduction in the TJ proteins. The effects of urea were dramatically amplified by urease which caused cell detachment, dissipation of transepithelial electrical resistance, and massive loss of the TJ proteins [16]. These experiments documented, for the first time, that uremia-induced disruption of intestinal barrier function is, in part, mediated by urea which has heretofore been considered to be a nontoxic retained metabolite. These findings revealed a novel mechanism for the previously documented salutary effect of urea-lowering strategies, e.g. low protein diet and longer and more frequent dialysis regimens in advanced CKD.

Activated charcoal has been widely used as a decontaminant in cases of acute poisoning and as an effective degassing agent to reduce abdominal bloating and flatulence. In addition, AST-120, a highly potent activated charcoal preparation has been shown to markedly reduce plasma concentration of indoxyl sulfate and p-cresol sulfate $[17,18]$ which are well known uremic toxins produced by the gut microbial flora [19-21]. Moreover administration of AST-120 in animal models of chronic re- 
nal disease has been shown to reduce oxidative stress and inflammation and retard progression of renal disease [22-24]. The salutary effects of AST-120 have been primarily attributed to its ability to limit formation and absorption of indoxyl sulfate and p-cresol sulfate $[25,26]$. As noted above, our recent study demonstrated the role of urea-derived ammonia and ammonium hydroxide as a major cause of the uremia-induced intestinal epithelial barrier destruction/dysfunction [16]. Given the ability of activated charcoal to avidly adsorb ammonia, AST-120 can potentially attenuate the severity of uremia-induced intestinal barrier disruption. If true, the widely reported salutary effects of AST-120 in reducing inflammation and oxidative stress may be, in part, due the preservation of intestinal epithelial barrier function and structure. The present study was undertaken to test the hypothesis that administration of AST-120 may attenuate uremia-induced disruption of intestinal epithelial TJ apparatus in animals with chronic renal failure.

\section{Methods}

\section{Animals}

Male Sprague-Dawley rats were purchased from Harlan Sprague Dawley Inc. (Indianapolis, Ind., USA). The rats were randomized to the control or the CKD group. The rats assigned to the control group were fed regular rat chow while those assigned to the CKD group were fed the rat chow containing $0.7 \%$ adenine for 2 weeks. The $\mathrm{CKD}$ rats were then randomized to receive either regular powdered rat chow or powdered rat chow containing AST-120 for additional 2 weeks. AST-120 (Mitsubishi Tanabe pharmaceutical Inc., Osaka, Japan) was given at a dose of $4 \mathrm{~g} / \mathrm{kg}$ body weight/day. Control animals were maintained on regular rat chow throughout the observation period. The animals were housed in a temperature-controlled facility with 12-hour light/dark cycles. Arterial blood pressure was determined by tail cuff plethysmography as described previously [27]. At the conclusion of the observation period, animals were placed in metabolic cages for a 24-hour urine collection. They were then anesthetized (ketamine/xylazine IP) and euthanized by exsanguination using cardiac puncture. The colon was removed and processed for expression of the key constituents of the TJ proteins by Western blot and immunohistological procedures. All experiments were approved by the University of California, Irvine, Institutional Committee for the Use and Care of Experimental Animals.

\section{Western Blot Analyses}

The tissues were homogenized on ice in modified RIPA lysis buffer containing $25 \mathrm{mM}$ Tris- $\mathrm{HCl} \mathrm{pH}$ 7.4, $150 \mathrm{mM} \mathrm{NaCl}, 1 \mathrm{mM}$ EDTA, 1\% NP-40, 0.1\% SDS, 1 mM PMSF and Protease Inhibitor Cocktail (Sigma-Aldrich, St. Louis, Mo., USA). Protein concentration in the tissue homogenates was determined by BSA assay kit (Pierce Rockford, Ill., USA), and $60 \mu \mathrm{g}$ of total protein from each sample was fractionated on 4-12\% Bis-Tris gradient gel (Invitrogen, Carlsbad, Calif., USA) at $120 \mathrm{~V}$ for $2 \mathrm{~h}$ and transferred to a Nitrocellulose Membrane. The membrane was then incubated with rabbit anti-claudin-1 or rabbit anti-occludin and mouse antiZO1 antibodies (Invitrogen) at 1:250 dilutions and anti-actin antibodies (Sigma-Aldrich) at 1:10,000 dilutions overnight. The appropriate horseradish peroxidase-conjugated secondary antibodies (Sigma-Aldrich) were used at a 1:5,000 dilution. The membrane was visualized with SuperSignal West Pico (Pierce) and developed by autoluminography.

\section{Immunohistochemical Procedures}

Paraffin tissue sections were deparaffinized with xylene, and antigens were unmasked using sodium citrate buffer in a highpower pressure cooker for $10 \mathrm{~min}$ and then cooled down to room temperature. Endogenous peroxidase activity was removed using $3 \%$ hydrogen peroxide in water, and blocked with Dako's Protein Block Serum-Free solution (Dako North America, Inc. Carpinteria, Calif., USA). The sections were then incubated overnight at $4^{\circ} \mathrm{C}$ with primary antibodies (1:50 or 1:80 rabbit anti-claudin- 1 or rabbit anti-occludin and mouse anti-ZO1; Invitrogen). Antibody binding was amplified using ImmPRESS ${ }^{\mathrm{TM}}$ Reagent anti-rabbit Ig or anti-mouse kit (Vector laboratories Inc., Burlingame, Calif., USA), and the complex visualized using diaminobenzidine. Nuclei were lightly stained with Mayer's hematoxylin.

Measurements of Selected Plasma Cytokine, Chemokine and Adhesion Molecules

The concentrations of IL- 6 , TNF- $\alpha$, MCP-1, macrophage inflammatory protein-3a (MIP-3a), cytokine-induced neutrophil chemoattractant-3 (CINC-3), chemokine ligand-5; CXCL5 (LIX), L-selectin, and intercellular adhesion molecule-1 (ICAM-1) in the pooled plasma samples of the study groups were measured using Rat Cytokine Array C2 kit (Ray Biotech Inc., Norcross, Ga., USA) following the manufacturer's instruction. The abundance of given proteins in the plasma was obtained as the dot signals on the membrane. Densitometry was performed, and the level of each cytokine was estimated against the corresponding standard. The data in the untreated and AST-120-treated groups were expressed as the percentage of the corresponding values found in the control group.

\section{Plasma Endotoxin Assay}

Plasma endotoxin level in the pooled samples was measured using Limulus Amebocyte Lysate Pyrogent kit (LONZA, Allendale, N.J., USA) following the manufacturer's instructions. All glassware and solutions used in the experiment were autoclaved. The plasma endotoxin levels are expressed as geometric mean end point $\log 10$ units/ml (EU/ml).

\section{Data Analysis}

Analysis of variance and Student's t test were used in the statistical evaluation of the data which are presented as mean \pm SD. $p$ values $<0.05$ were considered significant.

\section{Results}

\section{General Data}

Data are summarized in table 1. Compared to the control group, the untreated CKD rats exhibited significant 


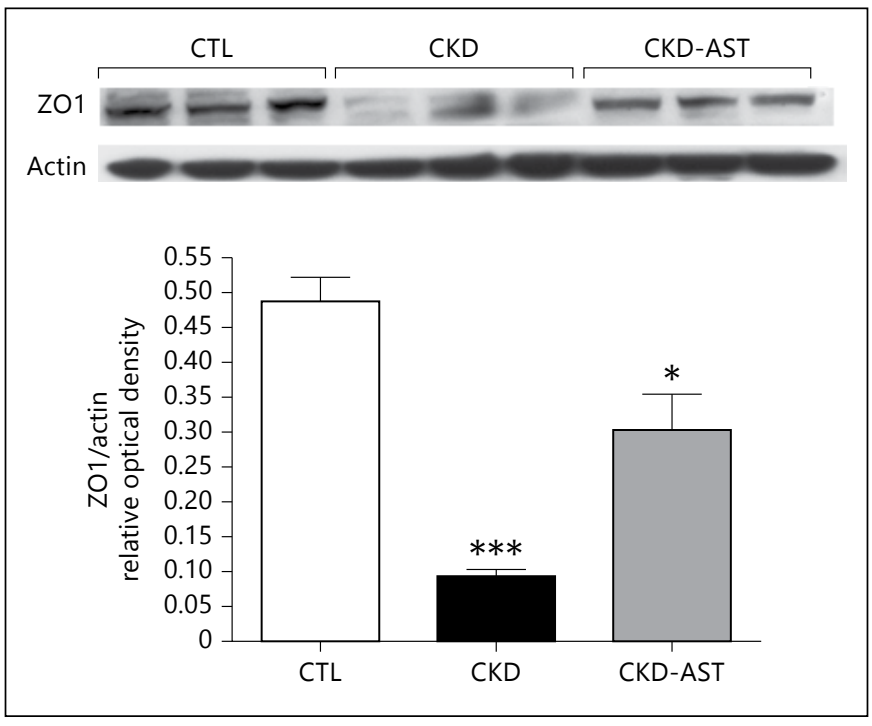

Fig. 1. Representative Western blot and group data depicting $\mathrm{ZO} 1$ protein abundance in colonic tissue from normal control (CTL), untreated CKD, and AST-120-treated CKD (CKD-AST) rats. $\mathrm{p}=0.0004, \mathrm{CTL}$ vs. $\mathrm{CKD} ; \mathrm{p}=0.0137, \mathrm{CKD}$ vs. CKD-AST.

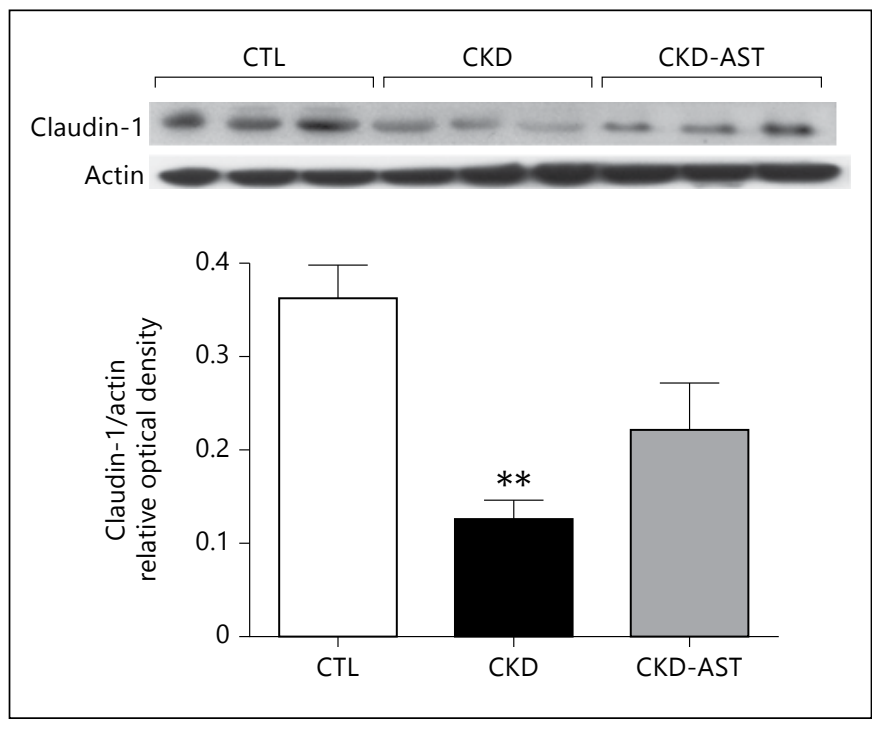

Fig. 2. Representative Western blot and group data depicting claudin-1 protein abundance in colonic tissue from CTL, untreated $\mathrm{CKD}$, and CKD-AST rats. $\mathrm{p}=0.0049$, CTL vs. CKD.

Table 1. General data of control, untreated CKD and AST-120-treated CKD groups

\begin{tabular}{|c|c|c|c|c|c|c|c|c|}
\hline Groups & $\begin{array}{l}\text { BW } \\
\mathrm{g}\end{array}$ & $\begin{array}{l}\text { U vol. } \\
\mathrm{ml}\end{array}$ & $\begin{array}{l}\mathrm{BP} \\
\mathrm{mm} \mathrm{Hg}\end{array}$ & $\begin{array}{l}\mathrm{Cr} \\
\mathrm{mg} / \mathrm{dl}\end{array}$ & $\begin{array}{l}\text { Urea } \\
\mathrm{mg} / \mathrm{dl}\end{array}$ & $\begin{array}{l}\text { Hct } \\
\%\end{array}$ & $\begin{array}{l}\text { MDA } \\
\mu \mathrm{M}\end{array}$ & $\begin{array}{l}\text { U protein } \\
\mathrm{mg} / 24 \mathrm{~h}\end{array}$ \\
\hline Control & $348 \pm 6.6$ & $7.6 \pm 1.6$ & $122 \pm 3.2$ & $0.42 \pm 0.05$ & $37.5 \pm 2.3$ & $47 \pm 0.9$ & $0.69 \pm 0.06$ & $17.4 \pm 2.4$ \\
\hline CKD-AST & $312 \pm 5.6^{*}$ & $29.3 \pm 1.4^{*}$ & $135 \pm 4.6^{\#}$ & $0.92 \pm 0.09^{*}, \#$ & $52.4 \pm 5.1^{*, \#}$ & $40 \pm 1.1^{*}$ & $1.07 \pm 0.10$ & $43.6 \pm 6.8^{*}$ \\
\hline
\end{tabular}

$\mathrm{BW}=$ Body weight; $\mathrm{U}$ vol. = urine volume; $\mathrm{BP}=$ systolic blood pressure; $\mathrm{Cr}=$ serum creatinine concentration; urea $=$ serum urea concentration; $\mathrm{Hct}=$ hematocrit; $\mathrm{MDA}=$ plasma malondialdehyde concentration; $\mathrm{U}$ protein $=$ urine protein excretion .

${ }^{*} \mathrm{p}<0.05$ vs. control; ${ }^{\#} \mathrm{p}<0.05$ vs. CKD.

reductions in body weight and hematocrit and significant increases in arterial pressure, urine protein excretion, and plasma creatinine and urea concentrations. This was associated with a marked increase in urine output reflecting impaired urinary concentrating capacity which is typical of chronic tubulointerstitial nephropathy in this model. Compared with the untreated CKD rats, the AST120 -treated group showed significantly lower arterial pressure, plasma urea and creatinine concentrations and higher hematocrit. The relative reduction in the plasma urea concentration $(38 \%)$ was far greater than that of creatinine (10\%) in the AST-120-treated compared to the untreated CKD group.

\section{TJ Protein Data}

Data are illustrated in figures 1-3. Compared with the control group, the untreated CKD group showed a marked reduction in protein abundance of the key transcellular TJ proteins, claudin-1 and occludin in the colonic tissue. The reduction in claudin-1 and occludin abundance in the untreated CKD rats was accompanied by marked $(\mathrm{p}<0.001)$ reduction in the main cytosolic plaque TJ protein ZO1. Consumption of AST-120 resulted in partial restoration of claudin-1, occludin and ZO1 in the colon of the treated CKD animals. The depletion of colonic tissue claudin-1, occludin, and ZO1 in the untreated CKD group and their partial restoration in the 


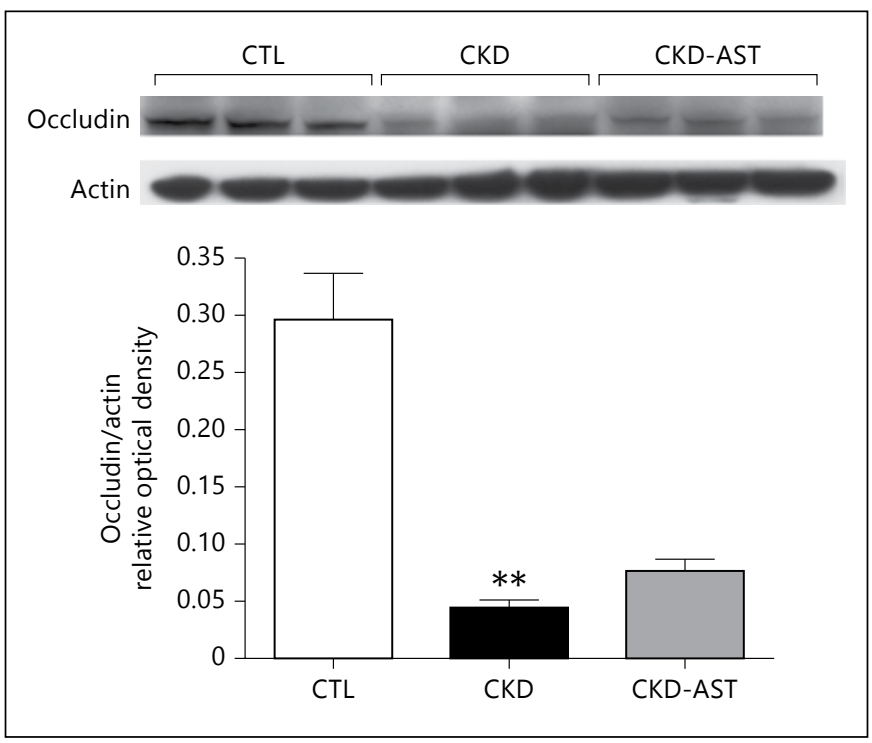

Fig. 3. Representative Western blot and group data depicting occludin protein abundance in colonic tissue from CTL, untreated $\mathrm{CKD}$, and CKD-AST rats. $\mathrm{p}=0.0037, \mathrm{CTL}$ vs. CKD.

AST-120-treated group found by Western blot analysis was confirmed by immunohistological examinations (fig. 4).

\section{Plasma Endotoxin Level}

No endotoxin was detected in the plasma samples from the normal control rats. In contrast, pooled plasma from the untreated CKD rats contained $1.4 \mathrm{EU} / \mathrm{ml}$ of endotoxin. Plasma endotoxin level in the AST-120-treated group $(0.7 \mathrm{EU} / \mathrm{ml})$ was $50 \%$ lower than that found in the untreated CKD group.

\section{Markers of Oxidative Stress and Inflammation}

Compared to the normal control rats, the untreated CKD animals showed a significant increase in the concentration of the plasma lipid peroxidation product malondialdehyde. AST-120 administration resulted in significant reduction of plasma malondialdehyde (table 1). These findings point to the presence of oxidative stress in CKD animals and its attenuation by AST-120. Compared with the control animals, the untreated CKD animals showed elevated plasma concentrations of inflammatory cytokines (IL-6 and TNF- $\alpha$ ), chemokines (MCP1 and CINC-3), chemokine ligands (MIP-3 $\alpha$ and LIX) and adhesion molecules (ICAM-1 and L-selectin). Administration of AST-120 reversed or markedly attenuated these abnormalities. These observations confirm the presence of systemic inflammation in untreated CKD rats and its attenuation with AST-120 therapy. Data are shown in figure 5 .

\section{Discussion}

Consumption of $0.7 \%$ adenine-containing diet has been shown to cause CKD by promoting severe crystalinduced chronic tubulointerstitial nephropathy [28]. As expected, the rats fed adenine-containing chow for 2 weeks exhibited significant elevation of plasma urea and creatinine concentrations, impaired urine-concentrating ability (polyuria), anemia, modest rise in blood pressure and mild proteinuria, consistent with the chronic interstitial nephropathy. In our previous study, we found that both 5/6 nephrectomy- and adenine-induced CKD resulted in an equally severe depletion of the colonic epithelial TJ proteins pointing to the causal role of CKD independent of its underlying etiology [14].

In confirmation of our original study [14], the CKD animals employed in the present study exhibited marked reductions in protein abundance of claudin- 1 and occludin, the key transcellular components of epithelial TJ, as well as ZO1 which is its main cytosolic component. This was associated with endotoxemia, elevation of plasma concentrations of inflammatory cytokines including IL-6 and TNF- $\alpha$, chemokines including MCP-1 and CINC-3 which promote infiltration of monocytes and neutrophilic granulocytes respectively, and adhesion molecules including ICAM-1 and L-selectin. Taken together, these findings suggest that disruption of the barrier structure of the colon, which is home to a huge community of microorganisms, contributes to the systemic inflammation by facilitating the entry of endotoxin and possibly other noxious compounds from the intestinal lumen to the internal milieu. This is compounded by our recent demonstration of CKD-induced profound alteration of the intestinal microbial flora in humans and experimental animals [29].

Administration of the activated charcoal preparation, AST-120, resulted in partial restoration of the TJ proteins in the treated animals. This was associated with significant reduction in serum urea and to a lesser extent of serum creatinine concentration. In addition, AST-120 administration reduced arterial pressure and lowered plasma concentration of malondialdehyde, pointing to attenuation of oxidative stress. As noted earlier, we have recently shown the central role of urea 
Fig. 4. Representative immunostaining photomicrographs depicting ZO1, occludin, and claudin-1 in colonic tissues from CTL, untreated CKD, and CKD-AST rats.
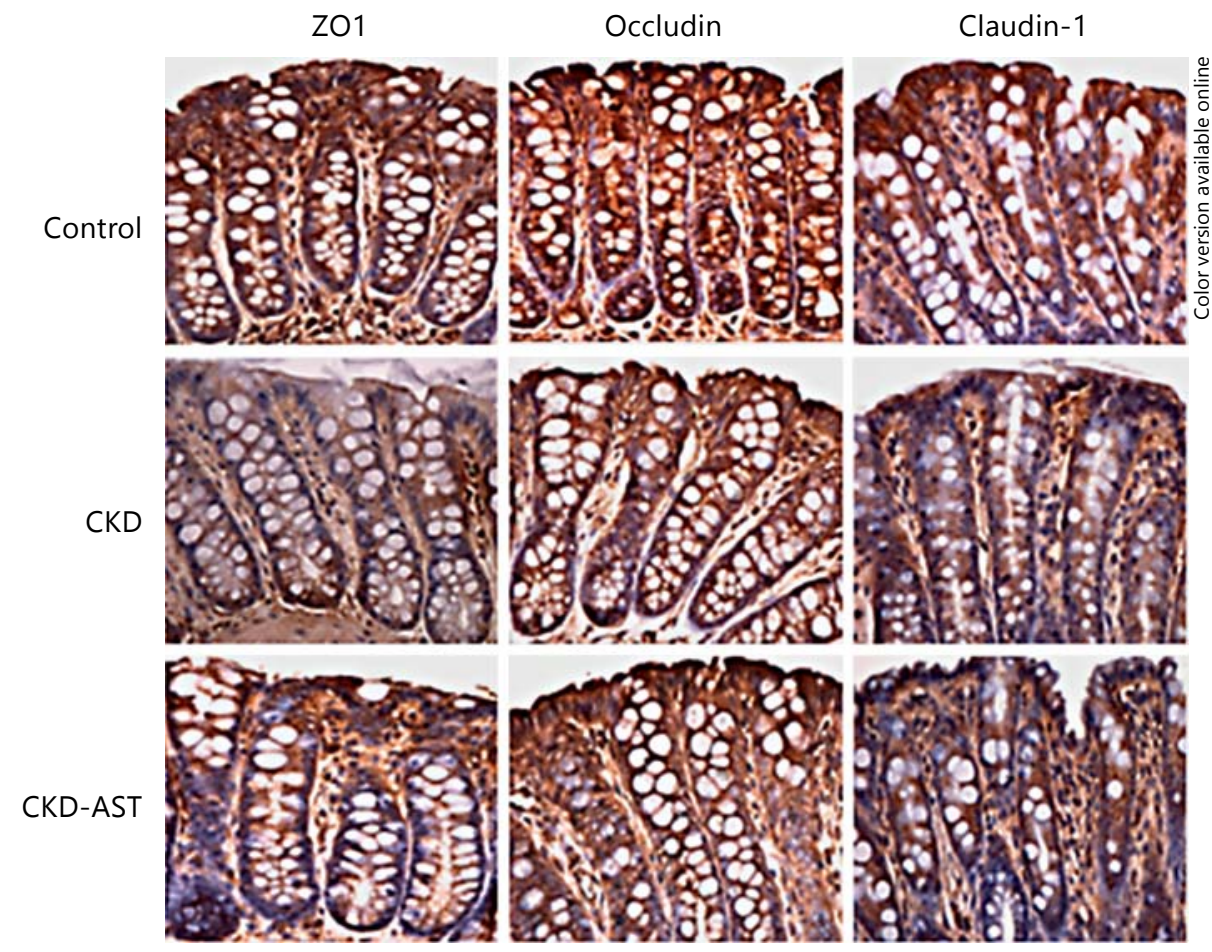

and the byproduct of its hydrolysis to ammonia by urease in the pathogenesis of uremia-induced breakdown of intestinal epithelial TJ and the resultant barrier dysfunction [16]. The activated charcoal sorbent AST-120 has been shown to markedly lower plasma concentration of indoxyl sulfate and p-cresol sulfate which are well known uremic toxins produced by the gut microbial flora, reduce oxidative stress and inflammation, and retard progression of CKD in experimental animals [22-24]. The beneficial effects of AST-120 have been primarily attributed to its ability to limit formation of indoxyl sulfate and $p$-cresol sulfate $[25,26]$. The present study demonstrated that AST-120 attenuates uremia-induced disruption of colonic epithelial TJ which has emerged as a major source of CKD-induced systemic inflammation. Thus, the widely reported salutary effects of AST-120 in reducing inflammation and oxidative stress are, in part, due the attenuation of uremia-induced intestinal epithelial barrier damage and dysfunction.

Compared to the untreated CKD rats, the AST120 -treated group showed a significant reduction in serum creatinine and urea concentrations. However, the magnitude of decline in urea level (35\%) was much greater than that of creatinine (14\%). While the reason for dif-

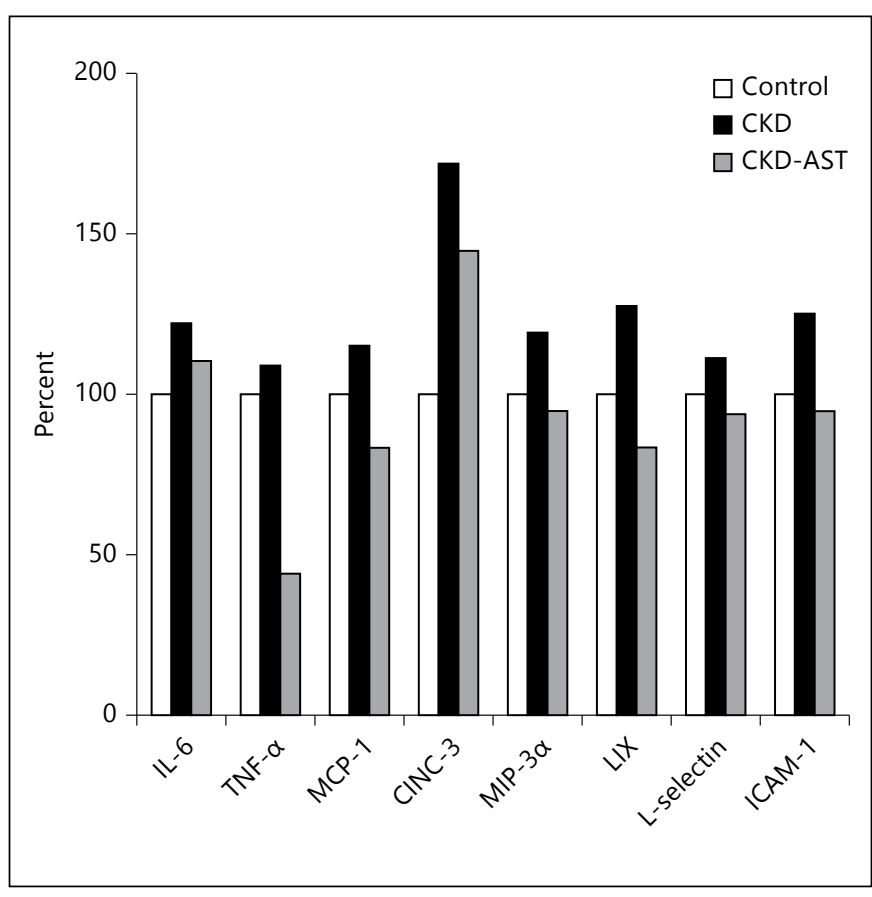

Fig. 5. Bar graphs depicting IL-6, TNF- $\alpha$, MCP-1, CINC-3, MIP$3 a$, LIX, L-selectin, and ICAM- 1 concentrations in the pooled plasma from CTL, untreated CKD, and CKD-AST rats. The data are presented as percent of values found in the pooled plasma from normal control rats. 
ferential effect of AST-120 on urea and creatinine levels is unclear, it may be due to the adsorption of urea or ureaderived ammonia by AST120 and hence reduced recycling of urea and de novo urea synthesis from ammonia in the liver.

Compared to the control group, untreated CKD rats showed endotoxemia and elevated plasma concentrations of several cytokines, chemokines, chemokine ligands, and adhesion molecules pointing to presence of systemic inflammation. Administration of AST-120 resulted in the reduction of plasma endotoxin and inflammatory cytokines, chemokines and adhesion molecules pointing to attenuation of systemic inflammation.

In addition to damaging the intestinal epithelial barrier structure and function, uremia been recently shown to result in profound alteration of the gut's microbial flora [29], which can further contribute to the systemic inflammation and uremic toxicity by generating harmful products $[6,20]$. The uremia-induced alteration of the intestinal microbial flora is in part driven by the influx of urea in the gastrointestinal tract and the associated modification of the luminal $\mathrm{PH}$ occasioned by formation of urea-derived ammonium hydroxide [6]. It is therefore possible that administration of oral activated charcoal may mitigate uremia-induced alteration of the gut microbiome as well. Future studies are planned to explore this possibility.

Taken together, the results of the present animal study suggest that oral activated charcoal adsorbent may be also effective in attenuating oxidative stress and inflammation which are the main cause of accelerated cardiovascular disease and numerous other complications in patients with end-stage renal disease. A properly conducted randomized placebo controlled clinical trial is needed to explore this possibility.

In conclusion, $\mathrm{CKD}$ animals exhibited depletion of the key protein constituents of the colonic epithelial TJ which was associated with endotoxemia, systemic inflammation, and oxidative stress. Administration of AST-120 attenuated uremia-induced disruption of colonic epithelial $\mathrm{TJ}$ and the associated endotoxemia, oxidative stress and inflammation.

\section{Acknowledgements}

This study was in part supported by grants from: NIH-NCRR UL1 TR000153, KL2 TR000147; the Juvenile Diabetes Research Foundation International 17-2011-609, and Mitsubishi Pharmaceutical Inc.

\section{References}

$>1$ Turner JR: Intestinal mucosal barrier function in health and disease. Nat Rev Immunol 2009;9:799-809.

-2 Nusrat A, Turner JR, Madara JL: Molecular physiology and pathophysiology of tight junctions. IV. Regulation of tight junctions by extracellular stimuli: nutrients, cytokines, and immune cells. Am J Physiol Gastrointest Liver Physiol 2000;279:G851-G857.

$\checkmark 3$ Cachofeiro V, Goicochea M, de Vinuesa SG, Oubiña P, Lahera V, Luño J: Oxidative stress and inflammation, a link between chronic kidney disease and cardiovascular disease. Kidney Int Suppl 2008;111:S4-S9.

$\checkmark 4$ Stenvinkel P: Inflammation in end-stage renal disease: the hidden enemy. Nephrology (Carlton) 2006;11:36-41.

$>5$ Ruiz S, Pergola PE, Zager RA, Vaziri ND: Targeting Nrf2 activation to ameliorate oxidative stress and inflammation in chronic kidney disease. Kidney Int, DOI: 10.1038/ ki.2012.439.

$\checkmark 6$ Vaziri ND: CKD impairs barrier function and alters microbial flora of the intestine - a major link to inflammation and uremic toxicity. Curr Opin Nephrol Hypertens 2012;21: 587-592.
7 Magnusson M, Magnusson KE, Sundqvist T, et al: Increased intestinal permeability to differently sized polyethylene glycols in uremic rats: effects of low- and high protein diets. Nephron 1990;56:306-311.

$>8$ Magnusson M, Magnusson KE, Sundqvist T, et al: Impaired intestinal barrier function measured by differently sized polyethylene glycols in patients with chronic renal failure. Gut 1991;32:754-759.

$>9$ de Almeida Duarte JB, de Aguilar-Nascimento JE, Nascimento M, et al: Bacterial translocation in experimental uremia. Urol Res 2004; 32:266-270.

10 Vaziri ND, Dure-Smith B, Miller R, et al: Pathology of gastrointestinal tract in chronic hemodialysis patients: an autopsy study of 78 cases. Am J Gastroenterol 1985;80:608611.

-11 Feroze U, Kalantar-Zadeh K, Sterling KA, Molnar MZ, Noori N, Benner D, Shah V, Dwivedi R, Becker K, Kovesdy CP, Raj DS: Examining associations of circulating endotoxin with nutritional status, inflammation, and mortality in hemodialysis patients. J Ren Nutr 2012;22:317-326.
12 Gonçalves S, Pecoits-Filho R, Perreto S, et al: Associations between renal function, volume status and endotoxaemia in chronic kidney disease patients. Nephrol Dial Transplant 2006; 21:2788-2794.

13 Szeto CC, Kwan BC, Chow KM, et al: Endotoxemia is related to systemic inflammation and atherosclerosis in peritoneal dialysis patients. Clin J Am Soc Nephrol 2008;3:431436.

14 Vaziri ND, Yuan J, Rahimi A, Ni Z, Said H, Subramanian VS: Disintegration of colonic epithelial tight junction in uremia: a likely cause of CKD-associated inflammation. Nephrol Dial Transplant 2012;27:26862693.

15 Vaziri ND, Goshtasby N, Yuan J, Jellbauer S, Moradi H, Raffatellu M, Kalantar-Zadeh K: Uremic human plasma degrades intestinal epithelial barrier structure and function. Am J Nephrol 2012;36:438-443.

16 Vaziri ND, Yuan J, Norris K: Role of urea in intestinal barrier dysfunction and disruption of epithelial tight junction in chronic kidney disease. Am J Nephrol 2013;37:1-6.

17 Goto S, Yoshiya K, Kita T, Fujii H, Fukagawa M: Uremic toxins and oral adsorbents. Ther Apher Dial 2011;15:132-134. 
18 Kikuchi K, Itoh Y, Tateoka R, Ezawa A, Murakami K, Niwa T: Metabolomic search for uremic toxins as indicators of the effect of an oral sorbent AST-120 by liquid chromatography/ tandem mass spectrometry. J Chromatogr B Analyt Technol Biomed Life Sci 2010;878: 2997-3002.

19 Meyer TW, Hostetter TH: Uremic solutes from colon microbes. Kidney Int 2012;81: 949-954.

20 Aronov PA, Luo FJ, Plummer NS, Quan Z, Holmes S, Hostetter TH, Meyer TW: Colonic contribution to uremic solutes. J Am Soc Nephrol 2011;22:1769-1776.

21 Niwa T: Recent progress in the analysis of uremic toxins by mass spectrometry. J Chromatogr B Analyt Technol Biomed Life Sci 2009;877:2600-2606
22 Bolati D, Shimizu H, Niwa T: AST-120 ameliorates epithelial-to-mesenchymal transition and interstitial fibrosis in the kidneys of chronic kidney disease rats. J Ren Nutr 2012; 22:176-180.

23 Shibahara H, Shibahara N: Cardiorenal protective effect of the oral uremic toxin absorbent AST-120 in chronic heart disease patients with moderate CKD. J Nephrol 2010; 23:535-540.

24 Nakamura T, Sato E, Fujiwara N, Kawagoe Y, Suzuki T, Ueda Y, Yamagishi S: Oral adsorbent AST-120 ameliorates tubular injury in chronic renal failure patients by reducing proteinuria and oxidative stress generation. Metabolism 2011;60:260-264.

25 Ito S, Higuchi Y, Yagi Y, Nishijima F, Yamato $\mathrm{H}$, Ishii $\mathrm{H}$, Osaka M, Yoshida M: Reduction of indoxyl sulfate by AST-120 attenuates monocyte inflammation related to chronic kidney disease. J Leukoc Biol 2013, Epub ahead of print.
26 Niwa T: Role of indoxyl sulfate in the progression of chronic kidney disease and cardiovascular disease: experimental and clinical effects of oral sorbent AST-120. Ther Apher Dial 2011;15:120-124.

27 Vaziri ND, Ni Z, Oveisi F, et al: Effect of antioxidant therapy on blood pressure and NO synthase expression in hypertensive rats. Hypertension 2000;36:957-964.

28 Yokozawa T, Zheng PD, Oura H and Koizumi F: Animal model of adenine-induced chronic renal failure in rats. Nephron 1986;44:230234.

29 Vaziri ND, Wong J, Pahl MV, Piceno YM, Yuan J, De Santis TZ, Ni Z, Nguyen TH, Andersen GL: Chronic kidney disease alters the composition of intestinal microbial flora. Kidney Int 2013;83:308-315. 